\title{
Eclipse retinopathy injury scale (ERIS): A classification of acute macular damage resulting from unprotected solar eclipse viewing
}

Maciej Czepita', Edyta Podlewska-Gaca², Damian Czepita ${ }^{1}$

${ }^{1}$ Private Eye Practice, Szczecin, Poland

${ }^{2}$ Adam Mickiewicz University, Astronomical Observatory Institute, Faculty of Physics, Poznań, Poland

Abstract

Introduction. Eclipse retinopathy occurs due to unprotected viewing of a solar eclipse. It is a long-recognized condition. The damage inflicted to the macula is due to a photochemical and photothermal effect caused by sunlight that enters the eye and is focused by the crystalline lens. Animal studies into eclipse retinopathy have been previously carried out. Retinal irradiance levels leading to macular damage have been established in rabbits. Limited data from studies on primates are also available. However, the exact values for humans have not yet been established with confidence. Methods. Here we present a simple method for estimation of the retinal irradiance dose in humans and a classification of macular damage.

Results. As an example, the retinal irradiance dose of a theoretical patient observing the solar eclipse of March 20, 2015, is given along with the grade of macular damage according to the developed classification.

Discussion. The retinal irradiance values given in the classification are provisional for the time being. With more widespread use among ophthalmologists the developed classification should become useful for prognostic purposes.

Solar eclipse $\cdot$ retinopathy $\cdot$ classification

Received: 16.01.2021, Accepted: 17.08.2021

\section{Introduction}

Throughout history humans have observed solar eclipses with fascination. To this day archeological evidence has survived of ancient solar eclipse observations carried out in different parts of the world during the Bronze Age. Descriptions of these observations can be found, for example, in Sumerian seals from modern-day northeastern Iraq [1] and rock carvings in Sweden [2]. In antiquity the health risks associated with observing solar eclipses began to be understood. In the Phaedo, Plato quotes Socrates (470-399 B.C.) as saying the safest method of observing a solar eclipse is by looking at the image reflected by water or some other medium [3]. In modern times, the first scientifically correct description of ocular damage resulting from unprotected solar eclipse viewing can be attributed to Théophile Bonet (1620-1689), who noted a central scotoma as a result of a thermal burn to the macula. More research into the photothermal and photochemical effects of sunlight on the retina, mainly in animal models, were carried out throughout the 19th and 20th centuries.

In 1944 Eccles and Flynn conducted a series of experiments on rabbits to observe the thermal effects of direct exposure to sunlight, and most importantly, gather data concerning the minimum amount of heat required to produce retinal lesions [4]. By subjecting anesthized rabbits to direct sunlight, the authors found that an exposure rate 
of 40 calories $/ \mathrm{cm}^{2} / \mathrm{min}^{-1}$ does not produce a retinal lesion. However, exposure at a rate of 70 calories $/ \mathrm{cm}^{2} / \mathrm{min}^{-1}$ does cause a severe lesion after 2.5 minutes, a less severe lesion after 30 seconds, a lesion in $50 \%$ of the examined rabbits after 10 seconds, and no lesions after only 3 seconds of exposure. These results were confirmed in ophthalmoscopic and histological examinations.

In 1980 McKechnie and Foulds [5] conducted similar examinations on rabbits to define the type of retinal damage incurred in scanning electron microscopy. The authors did not expose the rabbits to direct sunlight, however. A broad-spectrum artificial light source $(400-1150 \mathrm{~nm})$ was used instead. An exposure rate of either $140 \mathrm{~mW} / \mathrm{cm}^{2}$ or $230 \mathrm{~mW} / \mathrm{cm}^{2}$ for a period of 1 hour was used. The irradiance rate of $140 \mathrm{~mW} / \mathrm{cm}^{2}$ inflicted fragmentation of photoreceptor outer segments, edema of the outer nuclear layer, and distention of the retinal pigment epithelium cells, all of which, however, were almost fully recovered in 4 weeks. The higher irradiance level $\left(230 \mathrm{~mW} / \mathrm{cm}^{2}\right)$ produced complete destruction of the photoreceptors and retinal pigment epithelium. The damage was irreversible.

In primates studies are limited. Friedman et al. [6] studied the effects of exposure to the light from a clinical indirect ophthalmoscope on the retina of an anesthized rhesus monkey. The monkey was exposed to the light from the ophthalmoscope with a retinal irradiance of $0.27 \mathrm{~W} / \mathrm{cm}^{2}$ for 15 minutes. The accumulated dose was $243 \mathrm{~J} / \mathrm{cm}^{2}$. At this dose the retina and retinal pigment epithelium were found to be irreversibly damaged. A similar experiment was carried out also by Parver et al. [7]. Two cynomolgous monkeys were exposed to the light of an operating microscope with an irradiance of $1.06 \mathrm{~W} / \mathrm{cm}^{2}$ for a total of 1 hour. The total delivered dose was $3816 \mathrm{~J} / \mathrm{cm}^{2}$. Severe changes in the macula occurred as a result, within the photoreceptors and retinal pigment epithelium.

Similar studies on humans cannot be conducted. Therefore, exact data on irradiance levels of sunlight and macular damage in humans is not available. However, a scanning electron microscopy study from a 65-year-old patient scheduled to undergo enucleation for a choroidal malignant melanoma has yielded signs of similar damage at the cellular level [8]. Prior to enucleation the patient volunteered to look at the sun with the healthy eye occluded for a period of 10 minutes. Afterwards the affected eye was enucleated.

Currently, the photochemical explanation has been much more widely accepted as the cause of macular damage in solar retinopathy. Photochemical damage is believed to arise when light is absorbed by a chromophore and this leads to formation of an electronically active molecule that undergoes chemical transformation itself or interacts with other molecules. These transformed molecules can induce formation of free radicals and singlet oxygen molecules [9]. Ultimately, free radical molecules can cause lipid peroxidation of polyunsaturated fatty acids which make up cellular membranes. It is believed that the membranes of outer segments of photoreceptors and neurosensory retina and retinal pigment epithelium are particularly targeted [10]. However, it seems more likely that photochemical damage in solar retinopathy is not solely responsible for macular damage. Photothermal effects may also enhance the damage sustained by the macula [11]. Therefore, macular damage may be caused by a combination of both.

A limitation in evaluating photochemical damage in patients with eclipse retinopathy is a lack of in vivo clinical methods of testing. The diagnosis of eclipse retinopathy is made based on findings in ophthalmoscopy, color fundus imaging, optical coherence tomography (OCT) [12], shortwavelength fundus autofluorescence (FAF) [13] or nearinfrared autofluorescence (NIR-AF) [14], and other imaging modalities.

In this paper we propose a novel classification of macular damage in acute eclipse retinopathy based on color fundus imaging and a calculation of the estimated exposure to sunlight (retinal irradiance). We have designated this new classification as the Eclipse Retinopathy Injury Scale (ERIS).

\section{Classification of solar eclipse types}

The solar eclipse is an event in the sky when a celestial body passes in front of the surface of the sun. In our solar system we can observe transits of Mercury, Venus, the moon or other minor bodies (e.g. asteroids or comets) across the disk of the sun. The most spectacular however, is the solar eclipse by the moon. The moon is about 400 times smaller than the sun, but it is also almost 400 times closer to the Earth than the sun. Thus, the angular sizes of both objects are similar.

Depending on the geometry, and taking into account the fact that the moon's orbit around Earth, and Earth's orbit around the sun, are not perfectly round, but have slightly elliptical shapes, we can have few types of eclipses: total eclipse, partial eclipse, annular eclipse, and a hybrid eclipse. A total eclipse occurs when the silhouette of the moon completely covers the sun, allowing observers to see the much fainter solar corona. An annular eclipse occurs when the visual size of the moon is smaller than that of the sun. Hence, we can see the bright ring of the sun surrounding the dark moon. This type of eclipse happens when the moon and sun are exactly in one line with the Earth. When the three mentioned bodies are not exactly in one line the partial eclipse can occur. In such a situation, part of the moon's disk obscures the sun. The hybrid eclipse is a rather rare event. It happens when at some points on Earth it appears as a total eclipse, and at some points as annular. Although the solar eclipse is always a fascinating to observers, astronomers 
warn about watching the event with the unprotected eye, and looking at the sun directly through a telescope or binoculars is completely forbidden. Even using simple sunglasses is not enough because they usually reduce visible light (emitted at wavelengths $400-720 \mathrm{~nm}$ ) but transmit a significant fraction of energy in the infrared spectrum, which can cause severe eye damage. Instead, when looking at the sun, special filters should be used which significantly reduce solar radiation in all wavelengths to a safe level.

\section{Estimated exposure to sunlight during solar eclipse view- ing}

In order to calculate the damage caused by exposure of an eye to electromagnetic radiation, we have calculated how much energy is delivered to the eye by solar radiation during the eclipse. First, we have to notice that solar irradiance (defined as the power per unit area received from the sun) at the distance of 1 astronomical unit (1 AU) is $S=1361 \mathrm{~W} / \mathrm{m}^{2}$ The $S$ is known as the solar constant, and $1 \mathrm{AU}$ is the mean distance from the Earth to the sun. The value of $S$ is defined outside the Earth's atmosphere, so in order to calculate the amount of energy at the surface of the Earth, we have to take into account the reduction of radiation by the atmosphere, and the eye medium itself. The attenuation in the power of the solar radiation is caused mainly by absorption, scattering, and reflection in the atmosphere. For precise calculation one should include also the local conditions such as the influence of clouds, air pollution, and water vapor. However, these features are strongly place- and time-dependent, so they are not taken into account here. The full description of the calculation of atmospheric effects including the local weather conditions can be found in Gutiérrez-Trashorrasa et al. (2017) [15]. In our calculations we assume a clear sky and take into account the effects of reduction of solar radiation by the atmosphere, the effects related to solar elevation, and the height above sea level. Such approximation is enough for our purpose, as we calculate the maximum amount of solar radiation entering the eye. The local atmospheric and weather conditions can additionally reduce the energy, but this effect is usually negligible during the solar eclipse (except dense clouds which make observations impossible).

If we assume the sun is at zenith, the solar irradiance at sea level is reduced by $30 \%$ mainly due to absorption and scattering (Honsberg and Bowden, 2019) [16]. Moreover, we know that the decreasing solar elevation also effects the solar irradiance as the radiation passing through the thicker layer of the atmosphere is additionally diminished. This effect is included in the so-called air mass $\left(A_{m}\right)$ and can be calculated as:

$$
A_{m}=\frac{1}{\cos (z)} \text { where }
$$

$z$ - is the zenith distance $\left(z=90^{\circ}-h\right)$ with $h$ being solar elevation.

On the other hand, the intensity of solar radiation increases with the height above sea level. Thus, according to Honsberg and Bowden (2019) the solar luminosity can be estimated as:

$$
L=1.1 S\left[(1-0.14 h) 0.7^{A_{m}^{0.678}}+0.14 h\right]
$$

Here, $S$ is the solar constant $\left(1361 \mathrm{~W} / \mathrm{m}^{2}\right)$, the factor 0.7 comes from the fact that 0.3 solar radiation is reduced by the atmosphere, $\mathrm{h}$ is height above the sea expressed in kilometers, and the power term 0.678 expresses the empirical fit to the observed data and takes into account the nonuniformities in the atmospheric layers. Calculating the total luminosity reaching the Earth surface we take also correction on diffuse sky radiation, which increases the luminosity by $10 \%$ (factor 1.1 in equation 1). Diffuse radiation is the solar radiation reaching the observer, that has been scattered from the direct solar beam by molecules or particulates in the atmosphere. During the solar eclipse the luminosity takes the form

$$
L_{o c c}=\left(1-O_{b}\right) L
$$

with $O_{b}$ being the obscurity of the solar eclipse. When the whole surface of the Sun is visible the Eq. 2 converts to Eq. 1.

Now we estimate that the retinal irradiance $E_{r}$ can be expressed as

$E_{r}=L_{o c c} t_{m}\left(\frac{d_{p}^{2}}{d_{i}^{2}}\right)$

Here $d_{p}$ is the diameter of pupil, $d_{i}$ is the diameter of focused image (taken from OCT), and $t_{m}$ is the transmittance of the eye media. According to estimations made by Boettner and Wolter (1962) [10] the total transmittance of eye media is $83.5 \%$.

Although astronomers DO NOT recommend observing the solar eclipse with the naked (unprotected) eye, it is still a very popular way of watching the event.

\section{Eclipse Retinopathy Injury Scale}

Based on the estimated retinal irradiance $E_{r}$ and the diameter of the foveal lesion in the macula, the damage can be graded into three categories. An example of a foveal lesion and how its diameter is measured can be seen in Figure 2. Points are assigned for both the estimated retinal irradiance $\left(E_{r}\right)$ and separately for the diameter of the foveal lesion as captured in the color fundus image. Next, the points are added and the grade is given. A score of 2 points equals grade 1. A score of 3 points equals grade 2. Each additional point equals one grade higher in the classification. This can be seen in Table 1. 
Let us calculate the estimated retinal irradiance in a theoretical patient who viewed the solar eclipse on March 20, 2015, without any protection. The patient observed the eclipse in the city of Szczecin in Poland at around 10:21 a.m. for an uninterrupted 30 seconds. The eclipse at this location was partial. Totality occurred first in the North Atlantic Ocean just off the southern tip of Greenland and then through the Norwegian Sea and onwards through Svalbard and afterwards finally over to the North Pole. The obscurity of the eclipse in Szczecin at 10:21 a.m. was $45.40 \%$. The pictures taken during the event can be seen in Fig. 1. The mean height above sea level is taken as $0.05 \mathrm{~km}$ $(50 \mathrm{~m})$. In the calculations we assumed the image diameter $d_{i}=0.15 \mathrm{~mm}$ and the pupil diameter $d_{p}=2 \mathrm{~mm}$. All results are presented in Table 2. For convenience we have included the retinal irradiance $E_{r}$ in commonly used SI units $\left(\mathrm{W} / \mathrm{m}^{2}\right)$ and for easier interpretation (cal/s/cm${ }^{2}$ ). According to Table 2 it can be seen that a one-second exposure at the deep phase of the occultation, where almost half of the surface of the sun is covered, gives retinal irradiance at level $1.66\left(\mathrm{cal} / \mathrm{s} / \mathrm{cm}^{2}\right)$, so even 30 seconds of direct exposure gives the total value of irradiance $\mathrm{E}_{\mathrm{r}_{\text {tot }}}=49.8 \mathrm{cal} / \mathrm{cm}^{2}$, which causes $150-200 \mu \mathrm{m}$ diameter of macular damage, and classifies it as grade 2 in our Eclipse Retinopathy Injury Scale.

\section{Discussion}

The estimated retinal irradiance values presented in this classification are extrapolated to humans from the studies carried out by Eccles and Flynn [4] on rabbits. The exact values for humans have not yet been established with confidence. Therefore, the values should be treated as provisional. However, it is logical to believe that in humans,

Table 1. Eclipse Retinopathy Injury Scale for the classification of acute macular damage caused by unprotected solar eclipse viewing

\begin{tabular}{|c|c|c|c|}
\hline score & calculated $\mathrm{E}_{\mathrm{r}}$ & diameter of macular damage & grade \\
\hline 1 point & $\mathrm{E}_{\mathrm{r}}=12-35 \mathrm{cal} \mathrm{cm}^{-2} \mathrm{~min}^{-1}$ & $100-150 \mu \mathrm{m}$ & 2 points - grade 1 \\
\hline 2 points & $\mathrm{E}_{\mathrm{r}}=35-140 \mathrm{cal} \mathrm{cm}^{-2} \mathrm{~min}^{-1}$ & $150-200 \mu \mathrm{m}$ & 3 points - grade 2 \\
\hline 3 points & $\mathrm{E}_{\mathrm{r}}>140 \mathrm{cal} \mathrm{cm}^{-2} \mathrm{~min}^{-1}$ & $>200 \mu \mathrm{m}$ & 4 points - grade 3 \\
\hline & & & 5 points - grade 4 \\
\hline
\end{tabular}
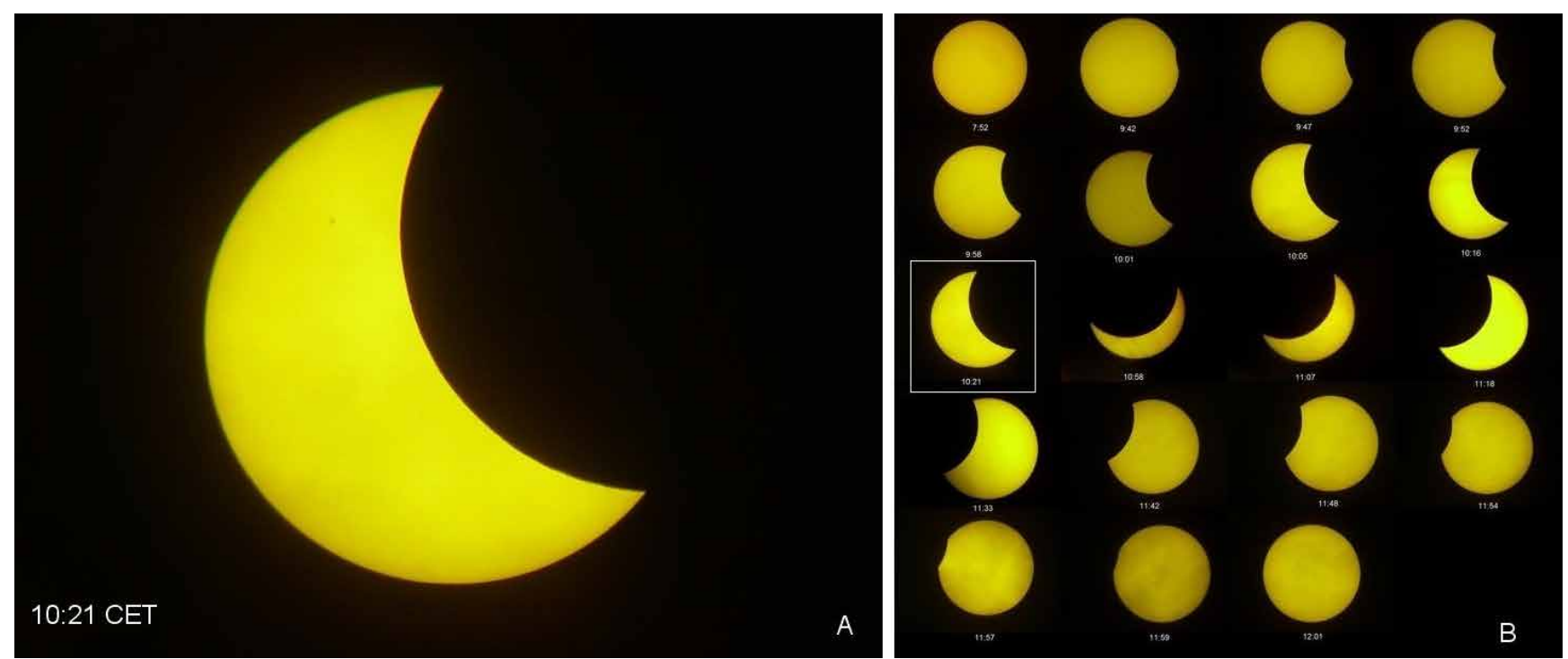

Figure 1: Photographs of the solar eclipse on March 20, 2015, taken with a $15 \mathrm{~cm}$ Newtonian reflector telescope by the first author (M.C.) from Szczecin, Poland. Image A was taken at 10:21 CET, almost midway into the eclipse. In B several individual images of the eclipse have been assembled together including Image A (white box). The obscurity of the eclipse was $45.40 \%$ at $10: 21$ CET. 
Table 2. Local conditions for Szczecin, Poland, during the solar eclipse on March 20, 2015

\begin{tabular}{|c|c|c|c|c|c|}
\hline $\begin{array}{l}\text { Time of obser- } \\
\text { vation (CET) }\end{array}$ & $\begin{array}{c}\text { Solar elevation } \\
\text { (deg) }\end{array}$ & $\begin{array}{c}\text { Obscurity } \\
(\%)\end{array}$ & $\underset{\left(W / m^{2}\right)}{\mathrm{L}}$ & $\begin{array}{l}E_{o c c} \\
\left(W / m^{2}\right)\end{array}$ & $\begin{array}{c}E_{o c c} \\
(\mathrm{cal} / \mathrm{s} / \\
\left.\mathrm{cm}^{2}\right)\end{array}$ \\
\hline $7: 52$ & 14.8 & 0 & 610 & 90551 & 2.16 \\
\hline $10: 21$ & 31.9 & 45.4 & 860 & 69704 & 1.66 \\
\hline 12.01 & 36.4 & 0 & 893 & 132560 & 3.17 \\
\hline
\end{tabular}

as in rabbits, there should be three categories of exposure. One category will consist of patients in which only some would develop a detectable macular lesion. The second group surely would consist of patients with well-defined macular lesions that would be reversible. Finally, at some retinal irradiance level (third category), macular lesions are irreversible. The presented method of calculation of the estimated retinal irradiance dose is simple. The variables needed for the calculation, such as the obscurity of the solar eclipse [17], the solar elevation, and the altitude of the site of observation, can all be accessed freely nowadays over the internet. Therefore, the level of accuracy achieved with this method should be fairly high. Most importantly, with accumulation of data from patients over time it may be possible to also use the developed classification for prognostic purposes in eclipse retinopathy patients.
It should be noted that color fundus imaging was chosen for this classification over other imaging modalities such as OCT, FAF, or NIR-AF for a number of reasons. The accessibility of color fundus imaging is still much higher than the accessibility of other methods mainly due to the cost of the equipment. Another reason for the selection of only one imaging modality are the different characteristics of each method. Short-wavelength fundus autofluorescence (FAF) is used to map the density of lipofuscin, the main fluorophore of the retinal pigment epithelium. In solar retinopathy, FAF images of the macula are very difficult to interpret. The macular damage is usually difficult to detect. Near-infrared fundus autofluorescence (NIR-AF) is used to map the density of melanin within the retinal pigment epithelium and choroid. In NIR-AF images macular damage is easily detectable in solar retinopathy (Fig. 2B). However, obtaining NIR-AF images is more difficult than obtaining short-wavelength fundus autofluorescence images. In some equipment, advanced image processing techniques such as image stacking must be used to create a single good quality image. Optical coherence tomography is widely used in ophthalmology nowadays. Imaging of all the layers that constitute the retina is attainable with current equipment. Measuring the macular damage dimensions is simple to perform. High precision measurements can be obtained if adaptive optics are additionally used. Unfortunately, the
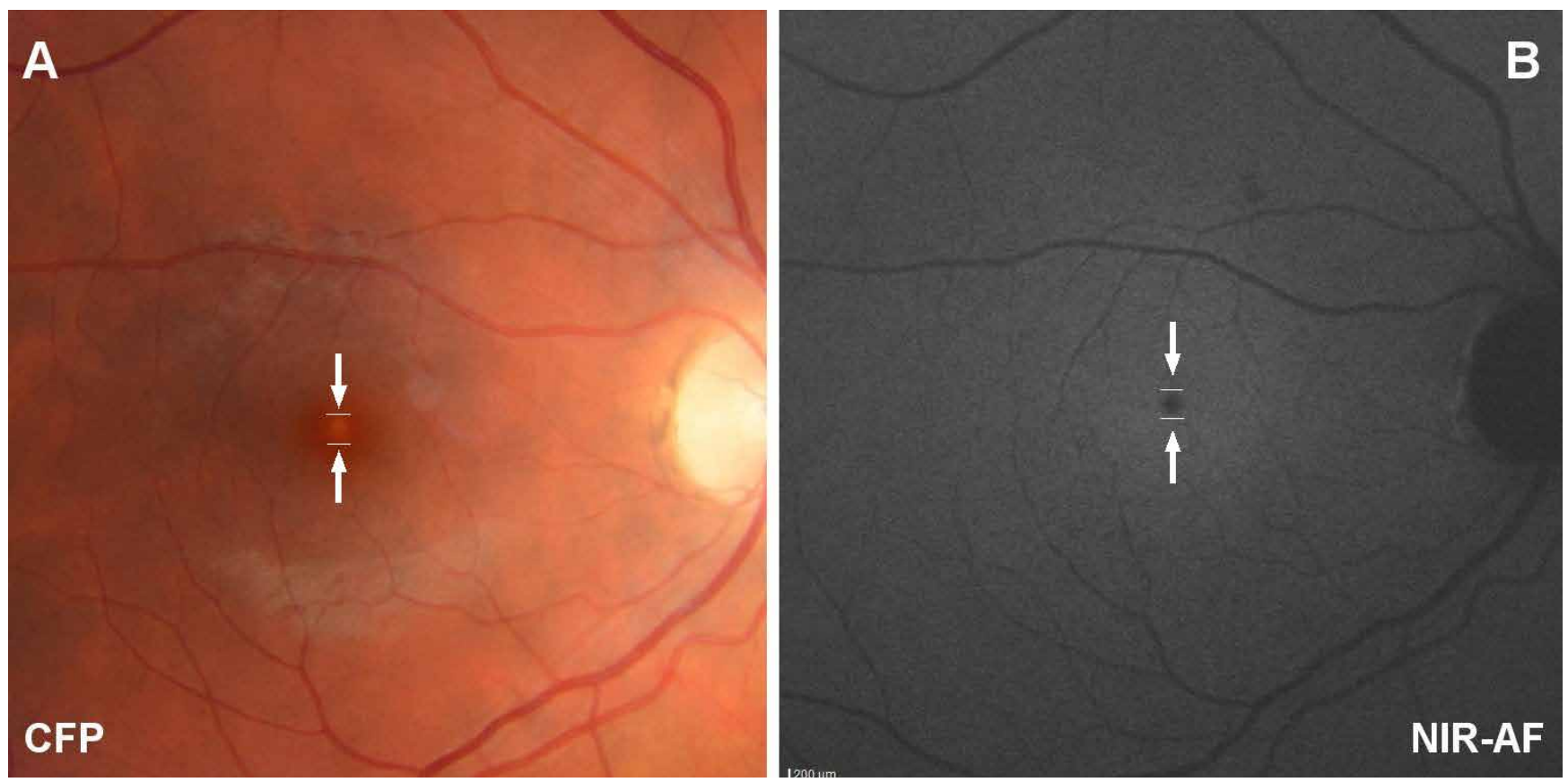

Figure 2. Color fundus photograph (CFP) of the right eye in a patient with solar retinopathy (A). Near-infrared fundus autofluorescence image (NIR-AF) of the right eye in the same patient (B). Macular damage diameter was measured (white arrows) in both images yielding a result of $139 \mu \mathrm{m}$ in the CFP image and $231 \mu \mathrm{m}$ in the NIR-AF image. 
cost of this equipment is still relatively high and therefore not always available, especially beyond larger hospitals. Color fundus photography is much more widespread. Typically in acute eclipse retinopathy a yellow foveal lesion in the macula develops, which is easily visible on ophthalmoscopy and in color fundus photographs [11]. Over time the lesion fades and is eventually replaced by a small lamellar hole.

A limitation to this method is a situation in which weather conditions during observations of the solar eclipse become variable. The total luminosity can be difficult to estimate. Therefore, the level of accuracy in the prediction may be affected. Another limitation is the application of this method only to acute cases of eclipse retinopathy. Estimation of the retinal irradiance dose in retrospect is very difficult. The foveal lesion observed in the macula undergoes change over time, making it impossible to reliably estimate the lesion's diameter at presentation. Nonetheless, the abovementioned fluctuations in total luminosity rarely occur and, regarding the time of presentation of a patient to the ophthalmologist, the majority of cases have been reported in the literature to occur in the acute phase.

\section{Conclusions}

The developed equations and classification are simple to use. The equipment needed is widely used and available even beyond a clinical setting. It is very important to gather a good account of the observations from the patient before performing the calculations. It should be expected that with accumulation of data from patients, it will be possible to use the developed classification for prognostic purposes in eclipse retinopathy patients.

\section{Authors' Contribution}

M.C.: Research concept and design, supervising the project, carrying out the experiments, acquisition of data, analysis and interpretation of data, designing the figures, drafting the article or revising it critically for important intellectual content, writing the manuscript, literature review, final proofreading, and approval of the version for publication; E.P.G.: Research concept and design, supervising the project, carrying out the experiments, acquisition of data, analysis and interpretation of data, designing the figures, drafting the article or revising it critically for important intellectual content, writing the manuscript, literature review, final proofreading, and approval of the version for publication; D.C.: Literature review

\section{Conflict of Interest}

The authors have no potential conflicts of interest to declare.

\section{Ethics Approval}

Due to the nature of the research, the consent of the ethics committee was not required.

\section{References}

[1] Morner N.A., Lind B.G. (2018). Astronomy and Sun cult in the Swedish Bronze Age. Int. J. Astron. Astrophys., 8: 143-162.

[2] Henriksson G. (2017). The acceleration of the Moon and the Universe - the mass of the gravitation. Adv. Astrophysics, 2017; 2 184-196.

[3] Michaelides M., Rajendram R., Marshall J., Keightley S. (2001). Eclipse retinopathy. Eye, 15: 148-151.

[4] Eccles J.C., Flynn A.J. (1943). Experimental photo-retinitis. Med. J. Aust., 16: 339-342.

[5] McKechnie N.M., Foulds W.S. (1980). Recovery of the rabbit retina after light damage (preliminary observations). Albrecht Von Graefes Arch. Clin. Exp. Ophthalmol., 212: 271-283.

[6] Friedman E., Kuwabara T. (1968). The retinal pigment epithelium. IV. The damaging effects of radiant energy. Arch. Ophthalmol., 80: 265-279.

[7] Parver L.M., Auker C.R., Fine B.S. (1983). Observations on monkey eyes exposed to light from an operating microscope. Ophthalmology, 90: 964-972.

[8] Hope-Ross M.W., Mahon G.J., Gardiner T.A., Archer D.B. (1993) Ultrastructural findings in solar retinopathy. Eye, 7: 29-33.

[9] Youssef P.N., Sheibani N., Albert D.M. (2011). Retinal light toxicity. Eye, 25: 1-14.

[10] Wu J., Seregard S., Algvere P.V. (2006). Photochemical damage of the retina. Surv. Ophthalmol., 51: 461-481.

[11] White T.J., Mainster M.A., Wilson P.W., Tips J.H. (1971). Chorioretinal temperature increases from solar observation. Bull. Math. Biophys., 33: 1-17.

[12] Wu C.Y., Jansen M.E., Andrade J., Chui T.Y.P., Do A.T., Rosen R.B., Deobhakta A. (2018). Acute solar retinopathy imaged with adaptive optics, optical coherence tomography angiography, and en face optical coherence tomography. JAMA Ophthalmol., 136 82-85.

[13] Merino-Suárez M.L., Belmonte-Martin J., Rodrigo-Auría F., Pérez-Cambrodí R.J., Piñero D.P. (2017). Optical coherence tomography and autofluoresceinography changes in solar retinopathy. Can. J. Ophthalmol., 52: e67-e71.

[14] Czepita M., Machalińska A., Czepita D. (2017). Near-infrared fundus autofluorescence imaging in solar retinopathy. GMS Ophthalmol. Cases, 7: Doc05. 
[15] Gutiérrez-Trashorras A.J., Villicaña-Ortiz E., Álvarez-Álvarez E., Gonzáles-Caballín J.M., Xiberta-Bernat J., Suarez-López M.J. (2018). Attenuation processes of solar radiation. Application to the quantification of direct and diffuse solar irradiances on horizontal surfaces in Mexico by means of an overall atmospheric transmittance. Renewable and Sustainable Energy Rev., 81: 93-106.

[16] Honsberg C.B., Bowden S.G. (2021). Photovoltaics Education Website. http://www.pveducation.org (13.01.2021).

[17] HM Nautical Almanac Office. (2021). Eclipse Online. http://astro. ukho.gov.uk/eclipse/ (13.01.2021). 